\title{
Textbook Outcome: an Ordered Composite Measure for Quality of Bariatric Surgery
}

\author{
Youri Q. M. Poelemeijer ${ }^{1,2}$ (D) Perla J. Marang-van de Mheen ${ }^{3} \cdot$ Michel W. J. M. Wouters $^{1,4} \cdot$ Simon W. Nienhuijs ${ }^{5}$. \\ Ronald S. L. Liem ${ }^{6,7}$
}

Published online: 19 December 2018

(C) The Author(s) 2018

\begin{abstract}
Introduction Textbook outcome (TO) studies have previously shown that a composite measure can provide additional information on the overall quality of surgical care. However, these were binominal outcomes which do not give individual hospitals the required information on how to improve their performance. The aim of this study is to create an ordered TO consisting of multiple outcome parameters for bariatric surgery to assess the extent of hospital variation.

Methods Patients who underwent a primary bariatric procedure in the Netherlands were included for analyses. The outcomes were ordered as mortality, severe postoperative complications, readmission, mild complications and prolonged length of stay (LOS) within 30 days after primary surgery with TO defined as none of these outcomes occurring. Hospitals were identified with a significantly higher or lower observed/expected ratio than expected based on case-mix and the extent of hospital variation was expressed as the median and interquartile range (IQR).

Results From a total of 27,360 patients on average, 88.7\% reached TO (range 35.5-96.9\%). Two hospitals had less than expected TO due to more prolonged LOS (57.6\%) in one hospital and more mild complications in another (17.1\%). Hospital variation was much smaller for TO (median OR 0.91 IQR [0.62-1.06]) than for an ordered TO (median POR 0.66 IQR [0.55-0.96]).

Conclusion Using the ordered TO for bariatric surgery, more hospital variation was captured thereby enabling individual hospitals to identify which outcomes and specific groups need improvement. This could attribute to the ongoing effort to improve the quality of the outcome of bariatric surgery.
\end{abstract}

Keywords Bariatric surgery · Gastric bypass $\cdot$ Sleeve gastrectomy $\cdot$ SG $\cdot$ RYGB $\cdot$ Textbook outcome (TO) $\cdot$ Netherlands $\cdot$ DICA . DATO $\cdot$ Dutch $\cdot$ Composite measure $\cdot$ Indicator $\cdot$ Audit $\cdot$ Registry

Electronic supplementary material The online version of this article (https://doi.org/10.1007/s11695-018-03642-1) contains supplementary material, which is available to authorized users.

Youri Q. M. Poelemeijer

Y.Q.M.Poelemeijer@lumc.nl

1 Scientific Bureau, Dutch Institute for Clinical Auditing, Leiden, Netherlands

2 Department of Surgery, Leiden University Medical Center, Albinusdreef 2, 2333 Leiden, ZA, Netherlands

3 Department of Biomedical Data Science, Leiden University Medical Center, Leiden, Netherlands

4 Department of Surgical Oncology, Netherlands Cancer Institute-Antoni van Leeuwenhoek Hospital, Amsterdam, Netherlands

5 Department of Surgery, Catharina Hospital, Eindhoven, Netherlands

6 Department of Surgery, Groene Hart Hospital, Gouda, Netherlands

7 Dutch Obesity Clinic, The Hague, Netherlands

\section{Introduction}

Several studies have shown that bariatric surgery can be considered safe, with a low postoperative mortality event rate of less than 1.0\%. [1-5] The results from the Dutch Audit for Treatment of Obesity (DATO) showed similar results. [6] Despite these positive results, between-hospital differences are still visible which offer opportunities for quality improvement. To achieve these improvements, several outcome indicators are established by DATO. [6] However, these outcome indicators provide insight into single outcome parameters, but do not necessarily provide insight into the entire care process in which different outcome parameters could be related to each other. [7, 8]

In the field of gastrointestinal cancer surgery and elective aneurysm surgery, a composite measure has been illustrated to give insight in the entire care process and make hospital 
comparison possible. [9-11] This composite measure has been described as textbook outcome (TO) and since TO covers the most desirable surgical outcomes, it gives a better impression of the overall quality of surgical care for the patient. [12-14] The indicator estimates the overall chance for a successful hospital admission and thus providing relevant information for the patient. In addition, event rates may be low for single outcome parameters, so that small differences between hospitals could be due to chance alone. Combining multiple outcome parameters provides more power to detect hospital differences and outcomes will be less likely different due to chance alone. [7] Ultimately, this results in a quality stimulus focused on all outcomes relevant for the patient and not only on single surgical outcome indicators.

Previous TO studies have shown that such a composite measure could provide additional information on the overall quality of surgical care from a patient's perspective. [9-11] However, the disadvantage of the earlier described TO indicator refers to combining all single outcome parameters into one binominal outcome. This binominal outcome does not give individual hospitals information where and how to improve if their performance is significantly worse than the national average. Ordering the different individual outcome parameters would make the composite measure more useful for quality improvement by professionals as well as suitable for the patient's perspective.

The aim of this study is to create a new ordered textbook outcome measure consisting of multiple postoperative outcome parameters for bariatric surgery and to assess whether this measure is more distinctive than individual parameters to estimate hospital differences.

\section{Methods}

\section{Patient Selection}

The study was designed as an observational study and unanimously approved by the scientific committee of DATO. Data were obtained from the national bariatric registry, a specific nationwide audit in which all 18 Dutch bariatric centres participate. [6] All patients undergoing a primary bariatric procedure between 1 January 2015 and 1 January 2018 were included for the analyses. Minimal data requirements were date of operation, type of surgery, bariatric technique and date of discharge. In addition, the parameters readmission, postoperative complications and mortality should be registered, up to 30 days after the primary surgery.

\section{Definitions}

Postoperative complications within 30 days after primary surgery were recorded by Clavien-Dindo Classification of
Surgical Complications (CD). [15] A mild complication was defined as CD-grade I or II and a severe complication was defined as CD-grade III or IV. Postoperative mortality, also registered as $\mathrm{CD}$-grade $\mathrm{V}$, was defined as mortality during the initial hospital stay or within 30 days after primary surgery.

Readmission was defined as the first readmission after discharge, but within 30 days after the initial intervention. A readmission is seen as a complication and therefore minimally marked as a mild complication. [16-18] Prolonged length of stay (LOS) was defined as discharge more than 2 days after primary procedure. $[19,20]$

\section{Parameters}

An ordinal composite outcome measure has been developed in previous research taking into account mutual relationships between mortality, readmission and prolonged length of stay. $[7,8]$ For the present study, members of the DATO scientific committee selected internationally described and relevant outcome parameters for desirable patient outcome after bariatric surgery. [17, 21-24] The measured outcome parameters were mortality, severe and mild postoperative complications, readmission and prolonged LOS, defined as hospital admission > 2 days after primary surgery.

\section{Ordering of Parameters}

Given possible relationships between the indicators, the hospital may be a positive outlier on one indicator and a negative outlier on another, thus requiring ordering to create an integral picture of quality of care. Ordering of individual parameters was based on expert advice and evidence from literature regarding what patients considered as better quality of care. [8] Previous research showed that patients considered complication after discharge (resulting in readmission) as worse quality of care compared to a complication during the same admission (resulting in prolonged LOS). [8, 25] Thereby, the ordering was defined as worst to best: mortality, severe complications, readmission, mild complications and prolonged LOS. Different combinations of these five quality indicators are possible within a single patient.

As readmission is considered as a mild complication, the combination of readmission and no mild complication is not possible. Similarly, a severe complication, which requires an intervention (CD-grade III) or intensive care observation (CD-grade IV) will exclude a normal LOS, unless this severe complication was the reason for a readmission. This results in 10 different groups in which all patients can be uniquely classified with the best group being similar to the textbook outcome in previous research (Online Supplement Table). 


\section{Validation}

The selection of parameters and ordering of the TO parameters were subsequently discussed in various forums, such as the Dutch national indicator days. During these days, the health insurers, national health care institute, patient federation and healthcare professionals meet to discuss the validity of different quality indicators. All parties have agreed to the proposed ordered parameters and official approval was given to continue and carry out the analyses.

\section{Analysis}

First, the percentage was calculated of patients for whom each individual outcome quality indicator was met. In addition, the proportion was calculated of patients for whom the conditional on all parameters listed above, in the ordered composite outcome, were met with the final (best) group being the TO. The group of patients is subsequently subdivided into one of the 10 ordered TO groups to assess the variation between hospitals in reasons why TO is not met, explaining which individual indicator is mainly responsible.

Second, a univariate logistic regression model was applied to study the associations between selected patient and procedure characteristics and achieving TO. The following characteristics were tested: age, gender, weight, height, BMI, procedure type (sleeve gastrectomy, gastric bypass or other procedure), type 2 diabetes mellitus (T2DM) (yes/no), hypertension (yes/no), dyslipidaemia (yes/no), gastro oesophageal reflex disease (GERD) (yes/no), obstructive sleep apnoea syndrome (OSAS) (yes/no) and musculoskeletal pain (yes/no). All variables with $p<0.05$ were included in the multivariate logistic regression analysis. Variables that remain independently associated with TO in multivariate analyses are relevant for fair hospital comparisons while considering potential differences in these patient and procedure characteristics.

Third, we estimated hospital differences in achieving TO using funnel plots with $95 \%$ control limits (C.I.). These plots were adjusted for those case-mix variables independently associated with TO in multivariate analyses. Casemix adjusted funnel plots show the actual observed (O) number of events divided by the expected (E) number of events on the $y$-axis $(\mathrm{O} / \mathrm{E}$ ratio). The expected (E) number of events is displayed on the $\mathrm{x}$-axis. A ratio greater than 1.0 indicates that more events have occurred than would have been expected based on case-mix of the hospital, while a ratio less than 1.0 indicates less events has occurred than would have been expected. We expressed the extent of hospital variation by calculating the median $\mathrm{O} / \mathrm{E}$ ratio with the interquartile range (IQR). The same was done for the ordered TO but using an ordinal logistic regression analysis to assess which case-mix variables were significantly associated (expressed as a proportional odds ratio (POR)).
Finally, to assess whether the ordered TO had better statistical properties in terms of identifying hospital differences, the relative efficiency of the TO versus each individual indicator was defined. To express the relative efficiency, the median SE of the coefficient of the hospital variable was used from a fixed effect logistic regression including hospital in addition to the statistically significant case-mix variables, as the SE reflects how precise hospital differences are estimated. This was done both for the ordered TO and the individual indicators and then compared to assess the efficiency of the ordered $\mathrm{TO}$ in relation to the individual indicators.

Analyses were performed using $\mathrm{R}$ version 3.5.1 in combination with the 'Companion to Applied Regression'-package (car 3.0-2), A Grammar of Data Manipulation'-package (dplyr 0.7.6), 'Tidy Data Functions'-package (tidyr 0.8.1), 'Table 1 Baseline Characteristics'-package (tableone 0.9.3), 'Convert Statistical Analysis Object'-package (broom 0.5.0) and 'Support Functions and Datasets'-package (MASS 7.3-50).

\section{Results}

A total of 27,360 unique patient records regarding primary bariatric surgery were entered by 18 Dutch hospitals. Twenty-seven thousand, two hundred seventy-three (27,273; $99.7 \%$ ) of these records contained complete data and were used for detailed analyses.

Table 1 shows the number and proportion of patients for whom each desired health outcome was realised. A total of $88.7 \%(n=24,201)$ patients reached TO after primary bariatric surgery. Looking at the differences between each conditional step in Table 1 mild postoperative complications (2.6\%; $n=720)$ and prolonged $\operatorname{LOS}(4.4 \% ; n=1182)$ had the greatest effect on achieving TO for the individual patient.

To gain insight into the variation between hospitals, each patient was assigned to one category of the ordered TO group.

Table 1 Population: percentage of patients for whom the outcome quality indicator was met. Conditional: percentage of patients for whom the outcome quality indicator, but also all outcome quality indicators listed above were met

\begin{tabular}{llllll}
\hline & \multicolumn{2}{l}{ Population } & & \multicolumn{2}{l}{ Conditional } \\
\cline { 2 - 3 } \cline { 6 - 7 } & $N$ & $\%$ & & $N$ & $\%$ \\
\hline Patients & 27,273 & & & \\
No mortality & 27,258 & $99.9 \%$ & & 27,258 & $99.9 \%$ \\
No severe complications & 26,573 & $97.4 \%$ & & 26,558 & $97.4 \%$ \\
No readmission & 26,534 & $97.3 \%$ & & 26,103 & $95.7 \%$ \\
No mild complications & 26,098 & $95.7 \%$ & & 25,383 & $93.1 \%$ \\
No prolonged LOS & 25,410 & $93.2 \%$ & 24,201 & $88.7 \%$ \\
Textbook outcome & & & & 24,201 & $88.7 \%$ \\
\hline
\end{tabular}




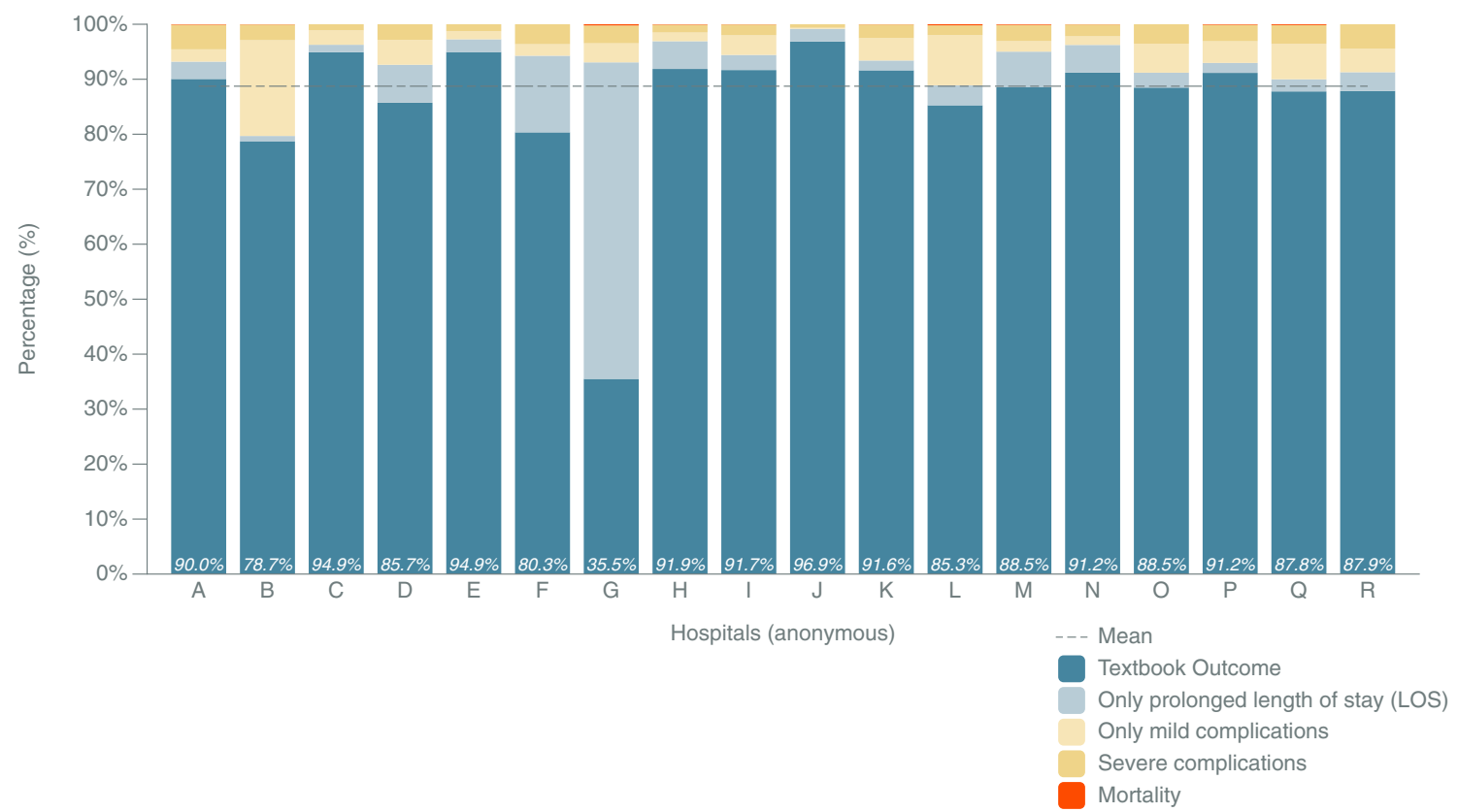

Fig. 1 Simplified view of ordered textbook outcome groups in bariatric surgery. Subcategories for mild complications and severe complications are combined into a general mild complications and severe complications category

The last group, the most favourable group, was defined as TO. Figure 1 shows a simple group classification and the variation between hospitals, with the emphasis on TO, prolonged LOS, mild complications, severe complications and mortality. On average, $4.3 \%$ (range $0.2 \%-17.1 \%$ between hospitals) of the patients had only a mild postoperative complication, and $2.6 \%$ (range $0.6 \%-4.5 \%$ ) had severe postoperative complications. It should be noted that the average for mild postoperative complications was heavily influenced by one hospital (B) that scored $17.1 \%$. In Fig. 1, the individual parameter 'readmission' is not included, because a readmission was by definition considered as a mild complication.

For a clear overview, Fig. 2 shows the distribution of parameters for not achieving TO. The figure is a detailed representation of all ordered TO groups of patients who have not achieved TO. The outlier of mild postoperative complications in Fig. 2 (hospital B) is visible. Another significant outlier is

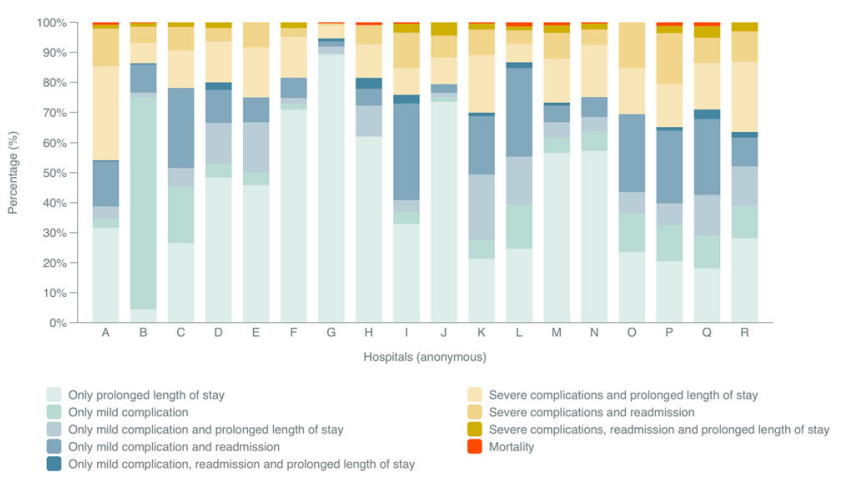

Fig. 2 Detailed view of ordered non-textbook outcome groups in bariatric surgery hospital $\mathrm{G}$ with $57.6 \%$ of the postoperative patients with a prolonged LOS. The national average was $4.2 \%$ (range $1.0 \%-57.6 \%$ ). In addition, particularly Fig. 2 shows the added value of the ordered TO groups above only having the binomial TO, thereby showing hospitals for which exact combination of outcomes they perform not as good as other hospitals do. For instance, patients with only a mild complication or readmission but with a normal LOS might be patients discharged too early and require a different type of intervention comparing to patients with also a prolonged LOS which might represent more complex patients.

\section{Impact of Patient and Procedure Characteristics}

Age, procedure type, T2DM, hypertension, dyslipidaemia, GERD and OSAS were factors associated with achieving TO in univariate logistic regression (Table 2). All factors remain significant in multivariate logistic regression, except for dyslipidaemia. Factors associated with a significantly effect on achieving TO were included in the case-mix model. Table 2 shows the same case-mix factors remaining significant for both the binomial and the ordered TO.

The variation between hospitals for the individual indicators is shown in Fig. 3 which again show the outlier hospitals $\mathrm{B}$ (on mild complications) and G (on prolonged LOS). It also shows that for each individual indicator, other hospitals scored significantly worse compared to the national average. A total of 12 out of 18 hospitals performed significantly worse on one or more single outcome parameters after case-mix correction, suggesting integration of these indicators in the ordered TO. 
Table 2 Patient characteristics

\begin{tabular}{|c|c|c|c|c|c|c|c|c|c|}
\hline & \multicolumn{2}{|c|}{ Non-textbook outcome } & \multicolumn{2}{|c|}{ Textbook outcome } & \multirow[b]{2}{*}{$p$ value } & \multicolumn{2}{|c|}{ Uni-/multi-variate logistic regression } & \multicolumn{2}{|c|}{ Ordinal logistic regression } \\
\hline & $N$ & $\%$ & $N$ & $\%$ & & OR & 95\% C.I. & OR & 95\% C.I. \\
\hline Number of patients & 3072 & $11.3 \%$ & 24,201 & $88.7 \%$ & - & - & - & - & - \\
\hline Age (mean, years, SD) & 45.0 & \pm 11.3 & 43.8 & \pm 11.3 & $<0.001$ & $0.99 *$ & $0.99-0.99$ & 0.99 & $0.99-1.00$ \\
\hline Gender (female) & 2407 & $78.4 \%$ & 19,281 & $79.7 \%$ & 0.092 & 1.08 & $0.99-1.19$ & - & - \\
\hline Weight (mean, kg, SD) & 126.3 & \pm 21.0 & 126.5 & \pm 20.2 & 0.549 & 1.00 & $1.00-1.00$ & - & - \\
\hline Length (mean, cm, SD) & 169.3 & \pm 9.2 & 169.4 & \pm 8.9 & 0.536 & 1.14 & $0.75-1.74$ & - & - \\
\hline BMI (mean, $\mathrm{kg} / \mathrm{m}^{2}, \mathrm{SD}$ ) & 44.0 & \pm 5.7 & 44.0 & \pm 5.5 & 0.855 & 1.00 & $0.99-1.01$ & - & - \\
\hline Sleeve gastrectomy & 822 & $26.8 \%$ & 5457 & $22.5 \%$ & - & REF & - & REF & - \\
\hline Gastric bypass & 2250 & $73.2 \%$ & 18,744 & $77.5 \%$ & $<0.001$ & $1.25^{*}$ & $1.15-1.37$ & 1.28 & $1.18-1.40$ \\
\hline $\mathrm{T} 2 \mathrm{DM}$ & 777 & $25.3 \%$ & 4803 & $19.8 \%$ & $<0.001$ & $0.73 *$ & $0.67-0.80$ & 0.82 & $0.74-0.90$ \\
\hline Hypertension & 1201 & $39.1 \%$ & 8150 & $33.7 \%$ & $<0.001$ & $0.79 *$ & $0.73-0.85$ & 0.90 & $0.82-0.98$ \\
\hline Dyslipidaemia & 695 & $22.6 \%$ & 4637 & $19.2 \%$ & $<0.001$ & 0.81 & $0.74-0.89$ & - & - \\
\hline GERD & 403 & $13.1 \%$ & 3079 & $12.7 \%$ & 0.555 & 0.97 & $0.86-1.08$ & - & - \\
\hline OSAS & 674 & $21.9 \%$ & 4361 & $18.0 \%$ & $<0.001$ & $0.78^{*}$ & $0.71-0.86$ & 0.85 & $0.77-0.94$ \\
\hline Musculoskeletal pain & 1425 & $46.4 \%$ & 10,944 & $45.2 \%$ & 0.229 & 0.95 & $0.88-1.03$ & - & - \\
\hline
\end{tabular}

$N$ number, $O R$ odds ratio, $S D$ standard deviation, $C I$ confidence intervals, T2DM type 2 diabetes mellitus, GERD gastroesophageal reflux disease, $O S A S$ obstructive sleep apnoea syndrome

* Factor remains significant after multivariate logistic regression and ordinal logistic regression analyses

The case-mix adjusted funnel plot for achieving TO shows the variation between hospitals (Fig. 4). Two hospitals scored significantly lower compared to the nationwide average and two hospitals had significant better scores. Comparing the extent of hospital variation in Figs. 3 and 4, the IQR is clearly smaller when using the $\mathrm{TO}$ and thus smaller hospital variation.
Fig. 3 Funnel plot of betweenhospital variation for a specific indicator after bariatric surgery (2015-2017). * O/E ratio: observed number of events divided by expected number of events. $* * \mathrm{O}=\mathrm{E}$ : the observed number equals the expected number of events. Expected based on population characteristics
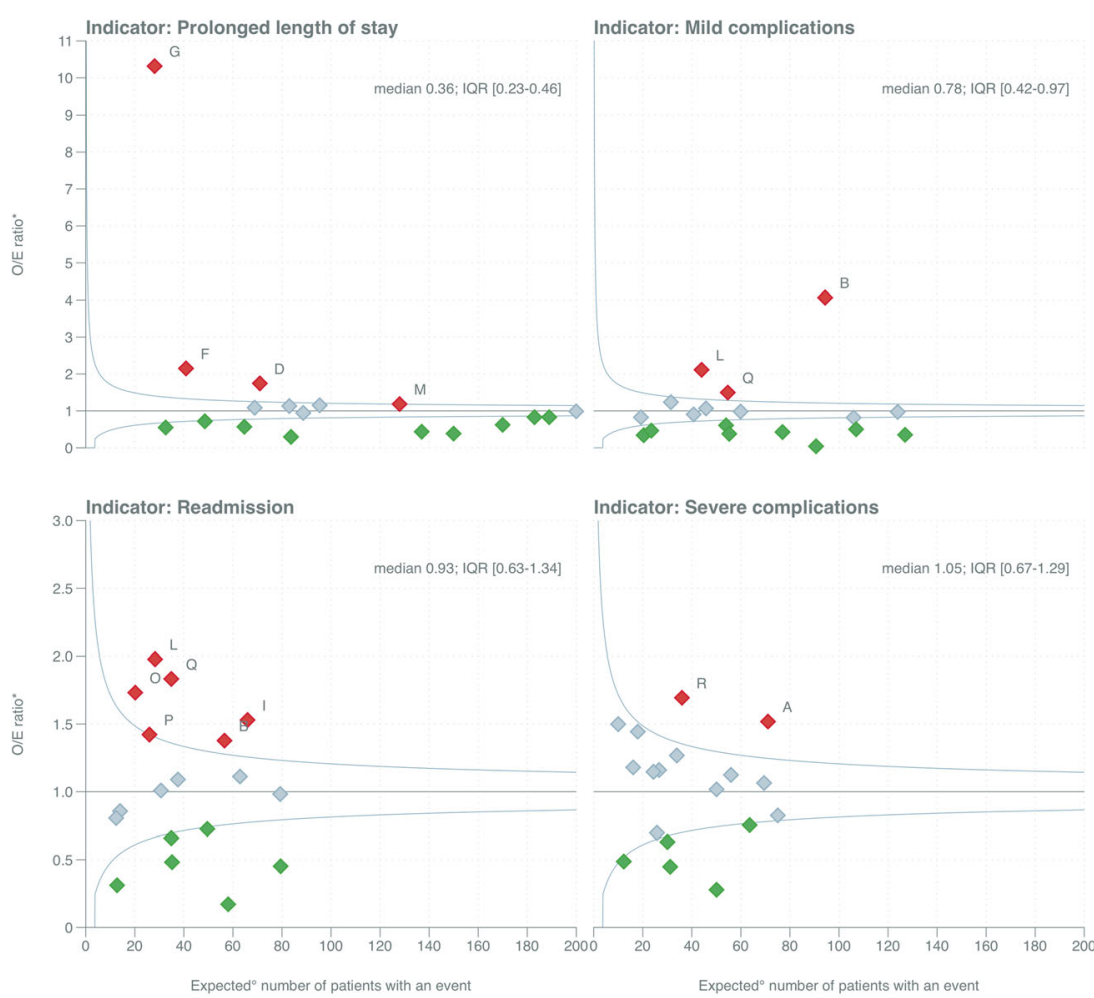

Indicator: Severe complications

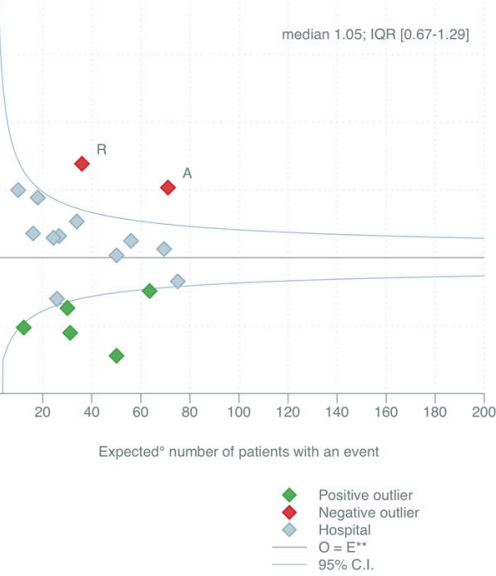




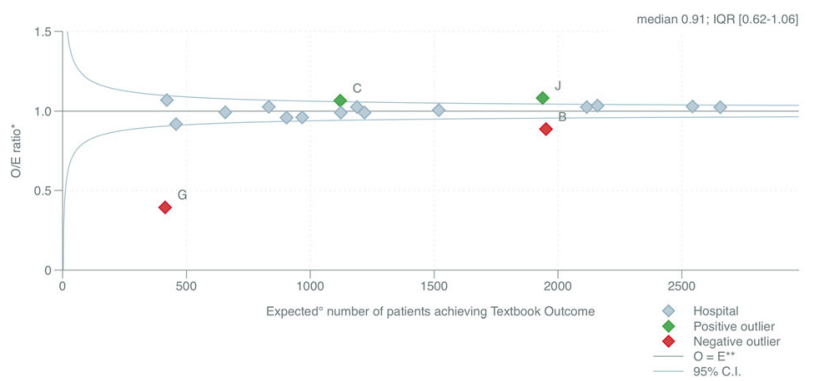

Fig. 4 Funnel plot of between-hospital variation in textbook outcome after bariatric surgery (2015-2017). " O/E ratio: observed number of achieved divided by expected number of textbook outcome. ${ }^{* *} \mathrm{O}=\mathrm{E}$ : the observed number equals the expected number of achieved textbook outcome. Expected based on population characteristics

In comparison, the ordered TO had a median 0.66 [IQR 0.55 $0.96]$, thereby showing the full extent of hospital variation which in part is cancelled out in the TO as different hospitals are outliers on the different indicators.

To express the relative efficiency of detecting hospital differences, Fig. 5 shows that using the ordered TO it is 11 times as likely to detect hospital differences when compared to mortality alone, about twice as likely compared to complications or readmissions and 1.2 times as likely compared to prolonged LOS. The relative efficiency of the ordered TO is comparable to that of the classic TO but with the advantage of capturing the full hospital variation as well as showing hospitals on which indicator they are an outlier.

\section{Discussion}

The present study uses existing previously developed composite outcome measure for other indications representing the percentage of patients with $\mathrm{TO}$ and added significant detail by ordering the different individual outcome parameters after primary bariatric surgery to make the TO more useful for quality improvement. The new composite measure (an ordered TO for bariatric surgery) still has the same properties as a composite measure for postoperative outcome quality

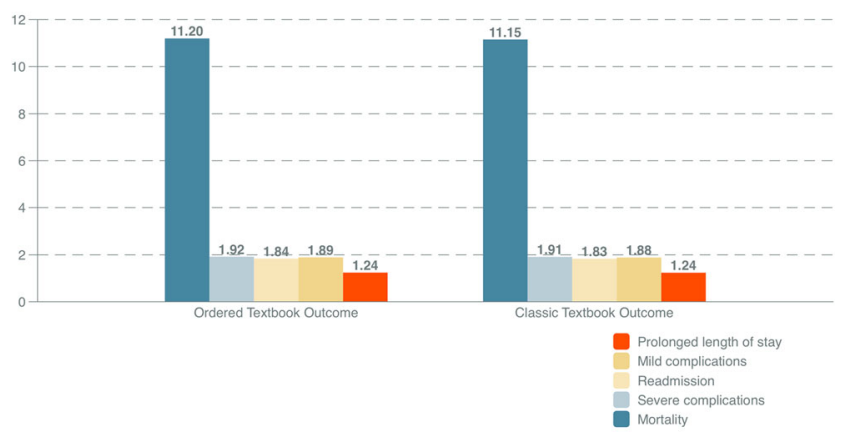

Fig. 5 Relative efficiency of two different textbook outcome indicators versus individual outcome indicators measurements, not only from a patient's perspective, but also providing more detailed information for the care giver.

It was shown, that for almost $90 \%$ of all primary bariatric patients, all desired health outcomes were realised. The binomial outcome indicator for achieving TO has smaller between-hospital variation as different hospitals were outliers for different indicators which cancelled out in the TO but were fully captured in the ordered TO. Besides, quality improvement of bariatric care has been primarily focused on mortality and morbidity rates alone and does not reflect the quality of care completely. [26-28] Thus, a hospital can score above average on a single indicator mortality, but score poorly on other processes regarding postoperative care like prolonged $\operatorname{LOS}[9,11,27]$ as was also seen in the present study. Another statistical pitfall is seen when the incidence and/or variation in mortality and morbidity is lacking, it hampers the discriminative ability of a single outcome indicator which is why either outcomes or multiple years are combined to improve this. [28, 29] Finally, outcomes parameters are often related to each other $[7,8$, $25]$ so that different hospitals are outliers on different indicators which are cancelled out in the dichotomous TO. Therefore, an ordered TO indicator was created to provide a tool in distinguishing hospitals, capturing the full extent of the hospital variation, and provide additional information for the individual care giver.

This ordered TO provides a good insight in different individual process indicators who influence the postoperative outcome. Because each patient can only be classified into one of the predetermined categories, there is a clear insight into the differences between hospitals for different combination of indicators thereby informing quality improvement initiatives. The ordered TO indicator identified hospitals who choose to hospitalise patients longer than the recommended 2 days. There were also hospitals that discharged their patients within 2 days after primary surgery but had a relatively higher percentage of readmissions related to a postoperative complication within 30 days after primary surgery. Despite the fact that the percentages differed little in achieving TO, there seem enough opportunities for hospitals to improve on individual indicators and their combinations given the variation.

Non-influential factors such as patient characteristics are often decisive for the type of procedure. [30] These factors can have an influence on achieving TO, while this has no direct relation with surgical expertise or team effort by the hospital. It is remarkable that the present study showed that a gastric bypass is associated with a higher likelihood (OR) of achieving TO compared to sleeve gastrectomy, while recent literature claims the opposite. [31, 32] This is most probably due to the large number of RYGB procedures in the Netherlands and the associated experience gained with this procedure. 


\section{Study Limitations}

Previous data verification by an independent third party showed no overall differences in patient, procedure and outcome data. [6] Therefore, it is unlikely that the results would have been influenced by the missing of almost $1 \%$ of the patient's data.

The present study indicated more than $10 \%$ of the primary operated patients did not achieve TO. The indicators 'mild complication' and 'prolonged LOS' were found to have the largest impact on achieving TO but were not associated with a reintervention or permanent morbidity. Therefore, these indicators may be considered as a 'minor morbidity' and it could be a point of discussion whether these indicators should be included in the definition of 'textbook outcome' as earlier discussed by Kolfschoten et al. [9] However, previous studies have shown that patients are willing to travel further for better quality of care. [33] Therefore, they were included in our suggested ordered TO for bariatric surgery.

At this moment, we have not applied a weighting for each parameter in this ordered TO, in the absence of evidence in the current literature of what the weight for each step or parameter should be. There is however evidence on ordering of the different parameters, which was the rationale of developing an ordered TO.

Another possible limitation of this study could be the absence of long-term follow-up results. However, long-term follow-up results reflect the quality of care given 5 years earlier. Short-term information reflects recently delivered care and is therefore more suitable and actionable for performance measures and inducing quality improvement cycles.

\section{Implications for the Future}

Now, the new indicator can be embedded in the current audit cycle, enhancing the insight of short-term postoperative complications per institute, whereby hospitals receive information on the short-term postoperative outcomes in a composite measure. In addition, different process parameters will be identified for each individual hospital in order to (further) improve the quality of care.

Furthermore, long (er)-term outcomes will have to be analysed to assess whether these are associated with the existing TO indicator. This will outline a completer picture of the full postoperative bariatric care process in each individual hospital.

\section{Conclusion}

An ordered textbook outcome for bariatric surgery is suggested as a composite measure for short-term postoperative outcome after bariatric surgery. Most importantly, individual hospitals can identify differences in outcome indicators using this ordered TO, whereas these may remain hidden in the previously developed binomial TO. This between-hospital variation may initiate an improvement cycle that will result in hospital and surgical quality improvements and therefore improve the clinical outcome of bariatric surgery.

\section{Compliance with Ethical Standards}

Conflict of Interest The authors declare that they have no conflict of interest.

Informed Consent For this type of study formal consent is not required.

OpenAccessThis article is distributed under the terms of the Creative Commons Attribution 4.0 International License (http://creativecommons.org/ licenses/by/4.0/), which permits unrestricted use, distribution, and reproduction in any medium, provided you give appropriate credit to the original author(s) and the source, provide a link to the Creative Commons license, and indicate if changes were made.

Publisher's Note Springer Nature remains neutral with regard to jurisdictional claims in published maps and institutional affiliations.

\section{References}

1. Welbourn R, Pournaras DJ, Dixon J, Higa K, Kinsman R, Ottosson $\mathrm{J}$, et al. Bariatric surgery worldwide: baseline demographic description and one-year outcomes from the Second IFSO Global Registry Report 2013-2015. Obes Surg. 2018;28(2):313-22.

2. Angrisani L, Santonicola A, Iovino P, et al. Bariatric surgery worldwide 2013. Obes Surg. 2015;25(10):1822-32.

3. Puzziferri N, Roshek 3rd TB, Mayo HG, et al. Long-term follow-up after bariatric surgery: a systematic review. JAMA. 2014;312(9): 934-42.

4. Hutter MM, Schirmer BD, Jones DB, et al. First report from the American College of Surgeons Bariatric Surgery Center Network: laparoscopic sleeve gastrectomy has morbidity and effectiveness positioned between the band and the bypass. Ann Surg. 2011;254(3):410-20. discussion 20-2

5. Buchwald H, Estok R, Fahrbach K, et al. Trends in mortality in bariatric surgery: a systematic review and meta-analysis. Surgery. 2007;142(4):621-32. discussion 32-5

6. Poelemeijer YQM, Liem RSL, Nienhuijs SW. A Dutch Nationwide Bariatric Quality Registry: DATO. Obes Surg. 2018;28(6):160210

7. Hofstede SN, van Bodegom-Vos L, Kringos DS, Steyerberg E, Marang-van de Mheen PJ. Mortality, readmission and length of stay have different relationships using hospital-level versus patient-level data: an example of the ecological fallacy affecting hospital performance indicators. BMJ Qual Saf 2018;27(6):474-83.

8. Lingsma HF, Bottle A, Middleton S, et al. Evaluation of hospital outcomes: the relation between length-of-stay, readmission, and mortality in a large international administrative database. BMC Health Serv Res. 2018;18(1):116.

9. Kolfschoten NE, Kievit J, Gooiker GA, et al. Focusing on desired outcomes of care after colon cancer resections; hospital variations in 'textbook outcome'. Eur J Surg Oncol. 2013;39(2):156-63.

10. Busweiler LA, Schouwenburg MG, van Berge Henegouwen MI, et al. Textbook outcome as a composite measure in oesophagogastric cancer surgery. Br J Surg. 2017;104(6):742-50. 
11. Karthaus EG, Lijftogt N, Busweiler LAD, Elsman BHP, Wouters M, Vahl AC, et al. Textbook outcome: a composite measure for quality of elective aneurysm surgery. Ann Surg. 2017;266(5):898904.

12. Dijs-Elsinga J, Otten W, Versluijs MM, et al. Choosing a hospital for surgery: the importance of information on quality of care. Med Decis Mak. 2010;30(5):544-55.

13. Jaarrapport (2016) Dutch Institute for Clinical Auditing; 2017 [082017]; Available from: https://dica.nl/jaarrapportage-2016.

14. Birkmeyer JD, Dimick JB, Staiger DO. Operative mortality and procedure volume as predictors of subsequent hospital performance. Ann Surg. 2006;243(3):411-7.

15. Dindo D, Demartines N, Clavien PA. Classification of surgical complications: a new proposal with evaluation in a cohort of 6336 patients and results of a survey. Ann Surg. 2004;240(2):205-13.

16. Abraham CR, Werter CR, Ata A, et al. Predictors of hospital readmission after bariatric surgery. J Am Coll Surg. 2015;221(1):220-7.

17. Lois AW, Frelich MJ, Sahr NA, et al. The relationship between duration of stay and readmissions in patients undergoing bariatric surgery. Surgery. 2015;158(2):501-7.

18. Dorman RB, Miller CJ, Leslie DB, et al. Risk for hospital readmission following bariatric surgery. PLoS One. 2012;7(3):e32506.

19. Carter J, Elliott S, Kaplan J, et al. Predictors of hospital stay following laparoscopic gastric bypass: analysis of 9,593 patients from the National Surgical Quality Improvement Program. Surg Obes Relat Dis. 2015;11(2):288-94.

20. Dogan K, Kraaij L, Aarts EO, et al. Fast-track bariatric surgery improves perioperative care and logistics compared to conventional care. Obes Surg. 2015;25(1):28-35.

21. Hopkins JC, Howes N, Chalmers K, et al. Outcome reporting in bariatric surgery: an in-depth analysis to inform the development of a core outcome set, the BARIACT Study. Obes Rev. 2015;16(1): 88-106.

22. Brethauer SA, Kim J, el Chaar M, et al. Standardized outcomes reporting in metabolic and bariatric surgery. Surg Obes Relat Dis. 2015;11(3):489-506.
23. Krell RW, Finks JF, English WJ, et al. Profiling hospitals on bariatric surgery quality: which outcomes are most reliable? J Am Coll Surg. 2014;219(4):725-34 e3.

24. Fischer C, Lingsma HF, Marang-van de Mheen PJ, et al. Is the readmission rate a valid quality indicator? A review of the evidence. PLoS One. 2014;9(11):e112282.

25. Marang-van de Mheen PJ, van Duijn-Bakker N, Kievit J. Surgical adverse outcomes and patients' evaluation of quality of care: inherent risk or reduced quality of care? Qual Saf Health Care. 2007;16(6):428-33.

26. Donabedian A. Evaluating the quality of medical care. 1966. Milbank Q. 2005;83(4):691-729.

27. Parina RP, Chang DC, Rose JA, et al. Is a low readmission rate indicative of a good hospital? J Am Coll Surg. 2015;220(2):169 76.

28. Dimick JB, Welch HG, Birkmeyer JD. Surgical mortality as an indicator of hospital quality: the problem with small sample size. JAMA. 2004;292(7):847-51.

29. Birkmeyer JD, Dimick JB, Birkmeyer NJ. Measuring the quality of surgical care: structure, process, or outcomes? J Am Coll Surg. 2004;198(4):626-32.

30. Bachler T, le Roux CW, Bueter M. How do patients' clinical phenotype and the physiological mechanisms of the operations impact the choice of bariatric procedure? Clin Exp Gastroenterol. 2016;9: 181-9.

31. Young MT, Gebhart A, Phelan MJ, et al. Use and outcomes of laparoscopic sleeve gastrectomy vs laparoscopic gastric bypass: analysis of the American College of Surgeons NSQIP. J Am Coll Surg. 2015;220(5):880-5.

32. Villamere J, Gebhart A, Vu S, et al. Body mass index is predictive of higher in-hospital mortality in patients undergoing laparoscopic gastric bypass but not laparoscopic sleeve gastrectomy or gastric banding. Am Surg. 2014;80(10):1039-43.

33. de Groot IB, Otten W, Smeets HJ, et al. Is the impact of hospital performance data greater in patients who have compared hospitals? BMC Health Serv Res. 2011;11:214. 